\title{
Common Fixed Point Theorems for Generalisation of R-Weak Commutativity
}

\author{
T. R. Vijayan, \\ Department of Mathematics, Pavai College of Technology, Namakkal -637018, India.
}

\begin{abstract}
The main purpose of this paper is to obtain fixed point theorems for $R$-weak commutativity which generalizes theorem 1 of R.P.Pant [2].
\end{abstract}

Key Words: and Phrases. Fixed point, coincidence point, compatible maps, non-compatible,

R-weak commuting maps.

\section{Introduction}

In 1986 Jungck [1] generalized the concept of weakly commuting mappings by introducing the notion of compatible maps. Since then the study of common fixed points of generalized contractions satisfying compatibility or some other commutativity conditions have emerged as an area of research activity. The central question concerning the common fixed points of generalized contractions may be formulated as given the self maps $\mathrm{A}_{\mathrm{i}}, \mathrm{B}_{\mathrm{i}}, \mathrm{S}_{\mathrm{i}}, \mathrm{T}_{\mathrm{i}} \forall \mathrm{i}$ of a metric space $(\mathrm{X}, \mathrm{d})$ satisfying a contractive condition what assumptions on commutativity and the contractive condition guarantee the existence of a common fixed point. For compatible maps satisfying the contractive condition.

(1) $\mathrm{d}\left(\mathrm{A}_{\mathrm{i}} \mathrm{x}, \mathrm{B}_{\mathrm{i}} \mathrm{y}\right)<\mathrm{M}_{\mathrm{ii}}(\mathrm{x}, \mathrm{y})=\max \left\{\mathrm{d}\left(\mathrm{S}_{\mathrm{i}} \mathrm{x}, \mathrm{T}_{\mathrm{i}} \mathrm{y}\right), \mathrm{d}\left(\mathrm{A}_{\mathrm{i}} \mathrm{x}, \mathrm{S} \mathrm{i}\right), \mathrm{d}\left(\mathrm{B}_{\mathrm{i}} \mathrm{y}, \mathrm{T}_{\mathrm{i}} \mathrm{y}\right),\left[\mathrm{d}\left(\mathrm{S}_{\mathrm{i}} \mathrm{x}, \mathrm{B}_{\mathrm{i}} \mathrm{y}\right)+\mathrm{d}\left(\mathrm{A}_{\mathrm{i}} \mathrm{x}, \mathrm{T}_{\mathrm{i}} \mathrm{y}\right)\right] / 2\right\} \forall{ }_{\mathrm{i}}$

(2) $d\left(A_{i} x, B_{i} y\right) \leq \phi\left(M_{i i}(x, y)\right)$ where $\phi: R_{+} \rightarrow R_{+}$is an upper semi-continuous function such that $\Phi(t)<t$, for each $\mathrm{t}>0$. And (3) there exists a function $\delta(0, \infty) \rightarrow(0, \infty)$, which is non decreasing or lower semi-continuous, such that $\in \leq \mathrm{M}_{\mathrm{ii}}(\mathrm{x}, \mathrm{y})<\in+\delta(\in)$ implies that $\mathrm{d}\left(\mathrm{A}_{\mathrm{i}} \mathrm{x}, \mathrm{B}_{\mathrm{i}} \mathrm{y}\right)<\in$.

Key Words and Phrases. Fixed point, coincidence point, compatible maps, non-compatible, R-weak commuting maps.

\section{Preliminaries}

Before proving our results, we need the following definitions and known results in this sequel.

Definition 2.1([2]).Two self maps $\mathrm{A}$ and $\mathrm{S}$ of a metric space(X,d) are called compatible if $\lim _{n \rightarrow \infty} d\left(A S_{x_{n}}, S A_{x_{n}}\right)=0$ whenever $\left\{\mathrm{x}_{\mathrm{n}}\right\}$ is a sequence in $\mathrm{X}$ such that $\lim _{n \rightarrow \infty} A x_{n}=\lim _{n \rightarrow \infty} S x_{n}=\mathrm{t}$ for some $\mathrm{t}$ in $X$.

Definition 2.2 ([2]). Two self maps A and $\mathrm{S}$ of a metric space(X, d) are defined to be R-weakly commuting at a point $\mathrm{x}$ in $\mathrm{X}$ if $\mathrm{d}\left(\mathrm{AS}_{\mathrm{x}}, \mathrm{SA}_{\mathrm{x}}\right) \leq \mathrm{Rd}\left(\mathrm{A}_{\mathrm{x}}, \mathrm{S}_{\mathrm{x}}\right)$ for some $\mathrm{R}>0$. The maps $\mathrm{A}$ and $\mathrm{S}$ are called point wise R-weakly commuting on $X$ if given $x$ in $X$ there exists $R>0$ such that $d\left(A S_{x}, S_{x}\right) \leq R d\left(A_{x}, S_{x}\right)$.

Remark 2.3.

- It is obvious that maps $\mathrm{A}$ and $\mathrm{S}$ are point wise R-weakly commuting on $\mathrm{X}<==>$ they commute at their coincidence points.

- If $\mathrm{A}$ and $\mathrm{S}$ commute at their coincidence, we can define $\mathrm{R}=\max \left\{1, \mathrm{~d}\left(\mathrm{AS}_{\mathrm{x}}, \mathrm{SA}_{\mathrm{x}}\right) / \mathrm{d}\left(\mathrm{A}_{\mathrm{x}}, \mathrm{S}_{\mathrm{x}}\right)\right\}$ when $\mathrm{A}_{\mathrm{x}} \neq \mathrm{S}_{\mathrm{x}}$, while $\mathrm{R}$ can be chosen arbitrarily when $\mathrm{x}$ is a coincidence point. The converse of this is obvious. Thus $\mathrm{A}$ and $\mathrm{S}$ can fail to be point wise R-weakly commuting only if they possess a coincidence point at which they do not commute.

- $\quad$ Compatible maps are necessarily point wise R-weakly commuting since compatible maps commute at their coincidence points.

R.P.Pant proved the following theorems.

Theorem 2.4 (R.P.Pant [1]).Let (A, S) and (B, T) be point wise R-weakly commuting pairs of self mappings of a metric space $(X, d)$ satisfying (i) $A X \subset T X, B X \subset S X$,

(ii) $d\left(A_{x}, B_{y}\right)<M(x, y)=\max \left\{d\left(S_{x}, T_{y}\right), d\left(A_{x}, S_{x}\right), d\left(B_{y}, T_{y}\right),\left[d\left(A_{x}, T_{y}\right)+d\left(B_{y}, S_{x}\right)\right] / 2\right\}$ whenever $M(x, y)>0$. Suppose that one of the pairs $(\mathrm{A}, \mathrm{S})$ or $(\mathrm{B}, \mathrm{T})$ is compatible and the other is Non compatible. If the mapping in the compatible pair be continuous then $\mathrm{A}, \mathrm{B}, \mathrm{S}$ and $\mathrm{T}$ have a unique common fixed point.

Theorem 2.5 (R.P.Pant [2]). Let $\left\{\mathrm{A}_{\mathrm{i}}\right\}, \mathrm{i}=1,2,3, \ldots . \mathrm{S}$ and $\mathrm{T}$ be self-mappings of a metric space (X, d) such that $A_{i} X \subset S X$ when $i>1, A_{1} X \subset T X$ and (i) Pairs $\left(A_{1}, S\right)$ and $\left(A_{i}, T\right), i>1$, are point wise R-weakly commuting with atleast one pair non compatible, 
(ii) $\mathrm{d}\left(\mathrm{A}_{1} \mathrm{x}, \mathrm{A}_{\mathrm{i}} \mathrm{y}\right)<\mathrm{M}_{1 \mathrm{i}}(\mathrm{x}, \mathrm{y})=\max \left\{\mathrm{d}\left(\mathrm{S}_{\mathrm{x}}, \mathrm{T}_{\mathrm{y}}\right), \mathrm{d}\left(\mathrm{A}_{1} \mathrm{x}, \mathrm{S}_{\mathrm{x}}\right), \mathrm{d}\left(\mathrm{A}_{\mathrm{i}} \mathrm{y}, \mathrm{T}_{\mathrm{y}}\right),\left[\mathrm{d}\left(\mathrm{A}_{1} \mathrm{x}, \mathrm{T}_{\mathrm{y}}\right)+\mathrm{d}\left(\mathrm{A}_{\mathrm{i}} \mathrm{y}, \mathrm{S}_{\mathrm{x}}\right)\right] / 2\right\}$.

Also let $\phi: \mathrm{R}_{+} \rightarrow \mathrm{R}_{+}$denote a function such that $\Phi(\mathrm{t})<\mathrm{t}$ for each $\mathrm{t}>0$. Whenever $\mathrm{M}_{1 \mathrm{i}}(\mathrm{x}, \mathrm{y})>0$ and $\mathrm{i}>1$.(iii)

$\mathrm{d}\left(\mathrm{A}_{1} \mathrm{x}, \mathrm{A}_{2} \mathrm{y}\right) \leq \phi\left(\mathrm{M}_{12}(\mathrm{x}, \mathrm{y})\right)$.If the range of one of the mappings is a complete subspace of $\mathrm{X}$ then all the $\mathrm{A}_{\mathrm{i}}, \mathrm{S}$ and $\mathrm{T} \forall \mathrm{i}$ have a unique common fixed point.

\section{Main Results}

In this section we prove common fixed point theorem for sequence of mappings that generalizes the theorem 2.5.

Theorem 3.1.Let $\left\{\mathrm{A}_{\mathrm{i}}\right\},\left\{\mathrm{B}_{\mathrm{i}}\right\},\left\{\mathrm{S}_{\mathrm{i}}\right\},\left\{\mathrm{T}_{\mathrm{i}}\right\} \forall \mathrm{i}=1,2,3, \ldots$ be self-mappings of a metric space (X, d) such that

$\mathrm{B}_{\mathrm{i}} \mathrm{X} \subset \mathrm{S}_{\mathrm{i}} \mathrm{X}, \mathrm{A}_{\mathrm{i}} \mathrm{X} \subset \mathrm{T}_{\mathrm{i}} \mathrm{X} \forall \mathrm{i}$ and (i) Pairs $\left(\mathrm{A}_{\mathrm{i}}, \mathrm{S}_{\mathrm{i}}\right)$ and $\left(\mathrm{B}_{\mathrm{i}}, \mathrm{T}_{\mathrm{i}}\right) \forall \mathrm{i}$ are Point wise R-weakly commuting with atleast one pair non compatible,

(ii) $\mathrm{d}\left(\mathrm{A}_{\mathrm{i}} \mathrm{x}, \mathrm{B}_{\mathrm{i}} \mathrm{y}\right)<\mathrm{M}_{\mathrm{ii}}(\mathrm{x}, \mathrm{y})=\max \left\{\mathrm{d}\left(\mathrm{S}_{\mathrm{i}} \mathrm{x}, \mathrm{T}_{\mathrm{i}} \mathrm{y}\right), \mathrm{d}\left(\mathrm{A}_{\mathrm{i}} \mathrm{x}, \mathrm{S}_{\mathrm{i}} \mathrm{x}\right), \mathrm{d}\left(\mathrm{B}_{\mathrm{i}} \mathrm{y}, \mathrm{T}_{\mathrm{i}} \mathrm{y}\right),\left[\mathrm{d}\left(\mathrm{S}_{\mathrm{i}} \mathrm{x}, \mathrm{B}_{\mathrm{i}} \mathrm{y}\right)+\mathrm{d}\left(\mathrm{A}_{\mathrm{i}} \mathrm{x}, \mathrm{T}_{\mathrm{i}} \mathrm{y}\right)\right] / 2\right\} \forall \mathrm{i}$ whenever $M_{i i}(x, y)>0$. If the range of one of the mappings is a Complete subspace of $X$ then all the $A_{i}, B_{i}, T_{i}$ and $S_{i} \forall i$ have a unique common fixe point.

Proof: Suppose that $\mathrm{T}_{\mathrm{i}}$ is non-compatible with $\mathrm{B}_{\mathrm{i}} \forall \mathrm{i}$.

Then there exists a sequence $\left\{\mathrm{z}_{\mathrm{n}}\right\}$ in $\mathrm{x}$ such that $\lim _{n \rightarrow \infty} B_{i} z_{n}=\lim _{n \rightarrow \infty} T_{i} z_{n}=\mathrm{t}$ for some $\mathrm{t}$ inX. $\forall \mathrm{i}$. But $\lim _{n \rightarrow \infty} d\left(B_{i} T_{i} z_{n}, T_{i} B_{i} z_{n}\right)$ is either non zero or does not exist. Since, $\mathrm{B}_{\mathrm{i}} \mathrm{X} \subset \mathrm{S}_{\mathrm{i}} \mathrm{X} \forall \mathrm{i}$ corresponding to each $\mathrm{z}_{\mathrm{n}}$ there exists $\mathrm{x}_{\mathrm{n}}$ in $\mathrm{X}$ such that $\mathrm{B}_{\mathrm{i}} \mathrm{z}_{\mathrm{n}}=\mathrm{S}_{\mathrm{i}} \mathrm{x}_{\mathrm{n}} \forall$ i. Thus $\mathrm{B}_{\mathrm{i}} \mathrm{Z}_{\mathrm{n}}=\mathrm{S}_{\mathrm{i}} \mathrm{x}_{\mathrm{n}} \rightarrow \mathrm{t}$ and $\mathrm{T}_{\mathrm{i}} \mathrm{z}_{\mathrm{n}} \rightarrow \mathrm{t}$ as $\mathrm{n} \rightarrow \infty$. We claim that $\mathrm{A}_{\mathrm{i}} \mathrm{x}_{\mathrm{n}} \rightarrow \mathrm{t}$ as $n \rightarrow \infty$.If not, then by virtue of (ii) for sufficiently large values of $n$ we get $d\left(A_{i} x_{n}, B_{i} Z_{n}\right) \leq M_{i i}\left(x_{n}, z_{n}\right)=M a x$ $\left\{\mathrm{d}\left(\mathrm{S}_{\mathrm{i}} \mathrm{x}_{\mathrm{n}}, \mathrm{T}_{\mathrm{i}} \mathrm{z}_{\mathrm{n}}\right), \mathrm{d}\left(\mathrm{A}_{\mathrm{i}} \mathrm{x}_{\mathrm{n}}, \mathrm{S}_{\mathrm{i}} \mathrm{x}_{\mathrm{n}}\right), \mathrm{d}\left(\mathrm{B}_{\mathrm{i}} \mathrm{Z}_{\mathrm{n}}, \mathrm{T}_{\mathrm{i}} \mathrm{Z}_{\mathrm{n}}\right),\left[\mathrm{d}\left(\mathrm{S}_{\mathrm{i}} \mathrm{x}_{\mathrm{n}}, \mathrm{B}_{\mathrm{i}} \mathrm{z}_{\mathrm{n}}\right)+\mathrm{d}\left(\mathrm{A}_{\mathrm{i}} \mathrm{x}_{\mathrm{n}}, \mathrm{T}_{\mathrm{i}} \mathrm{z}_{\mathrm{n}}\right)\right] / 2\right\} . \forall \mathrm{i}$.

$=\mathrm{d}\left(\mathrm{A}_{\mathrm{i}} \mathrm{x}_{\mathrm{n}}, \mathrm{S}_{\mathrm{i}} \mathrm{x}_{\mathrm{n}}\right)=\mathrm{d}\left(\mathrm{A}_{\mathrm{i}} \mathrm{x}_{\mathrm{n}}, \mathrm{B}_{\mathrm{i}} \mathrm{Z}_{\mathrm{n}}\right)$. Which is a contradication.

Hence $A_{i} X_{n} \rightarrow t$. Also, Since $A_{i} X \subset T_{i} X \forall i$

For each $\mathrm{x}_{\mathrm{n}}$ there exists $\mathrm{y}_{\mathrm{n}}$ in $\mathrm{X}$ such that $\mathrm{A}_{\mathrm{i}} \mathrm{x}_{\mathrm{n}}=\mathrm{T}_{\mathrm{i}} \mathrm{y}_{\mathrm{n}} \forall \mathrm{i}$ and $\mathrm{A}_{\mathrm{i}} \mathrm{x}_{\mathrm{n}}=\mathrm{T}_{\mathrm{i}} \mathrm{y}_{\mathrm{n}} \rightarrow \mathrm{t}$.

We show that $\mathrm{B}_{\mathrm{i}} \mathrm{y}_{\mathrm{n}} \rightarrow t \forall$. If not, then using (ii) for sufficiently large values of $\mathrm{n}$, we get $\mathrm{d}\left(\mathrm{A}_{\mathrm{i}} \mathrm{x}_{\mathrm{n}}, \mathrm{B}_{\mathrm{i}} \mathrm{y}_{\mathrm{n}}\right)<$ $\mathrm{M}_{\mathrm{ii}}\left(\mathrm{x}_{\mathrm{n}}, \mathrm{y}_{\mathrm{n}}\right)=\operatorname{Max}\left\{\mathrm{d}\left(\mathrm{S}_{\mathrm{i}} \mathrm{x}_{\mathrm{n}}, \mathrm{T}_{\mathrm{i}} \mathrm{y}_{\mathrm{n}}\right), \mathrm{d}\left(\mathrm{A}_{\mathrm{i}} \mathrm{x}_{\mathrm{n}}, \mathrm{S}_{\mathrm{i}} \mathrm{x}_{\mathrm{n}}\right), \mathrm{d}\left(\mathrm{B}_{\mathrm{i}} \mathrm{y}_{\mathrm{n}}, \mathrm{T}_{\mathrm{i}} \mathrm{y}_{\mathrm{n}}\right),\left[\mathrm{d}\left(\mathrm{S}_{\mathrm{i}} \mathrm{x}_{\mathrm{n}}, \mathrm{B}_{\mathrm{i}} \mathrm{y}_{\mathrm{n}}\right)+\mathrm{d}\left(\mathrm{A}_{\mathrm{i}} \mathrm{x}_{\mathrm{n}}, \mathrm{T}_{\mathrm{i}} \mathrm{y}_{\mathrm{n}}\right)\right] / 2\right\} . \forall \mathrm{i}$.

$=\mathrm{d}\left(\mathrm{A}_{\mathrm{i}} \mathrm{x}_{\mathrm{n}}, \mathrm{B}_{\mathrm{i}} \mathrm{y}_{\mathrm{n}}\right) \forall \mathrm{i}$ Which is a contradiction. Thus $\mathrm{A}_{\mathrm{i}} \mathrm{x}_{\mathrm{n}} \rightarrow \mathrm{t}, \mathrm{S}_{\mathrm{i}} \mathrm{x}_{\mathrm{n}} \rightarrow \mathrm{t}, \mathrm{T}_{\mathrm{i}} \mathrm{y}_{\mathrm{n}} \rightarrow \mathrm{t}, \mathrm{B}_{\mathrm{i}} \mathrm{y}_{\mathrm{n}} \rightarrow \mathrm{t} \forall \mathrm{i}$ where $\mathrm{T}_{\mathrm{i}} \mathrm{y}_{\mathrm{n}}=\mathrm{A}_{\mathrm{i}} \mathrm{x}_{\mathrm{n}} \forall \mathrm{i}$. Next, suppose that $\mathrm{S}_{\mathrm{i}} \forall \mathrm{i}$ is a noncompatible with $\mathrm{A}_{\mathrm{i}} \forall_{\mathrm{i}}$.

Then there exists a sequence $\left\{\mathrm{x}_{\mathrm{n}}\right\}$ in $\mathrm{X}$ such that $\lim _{n \rightarrow \infty} A_{i} x_{n}=\lim _{n \rightarrow \infty} S_{i} x_{n}=\mathrm{t}$ for some $\mathrm{t}$ in $\mathrm{X} . \forall \mathrm{i}$. But $\lim _{n \rightarrow \infty} d\left(A_{i} S_{i} x_{n}, S_{i} A_{i} x_{n}\right) \forall \mathrm{i}$ is either non zero or does not exist.

Since $\mathrm{A}_{\mathrm{i}} \mathrm{X} \subset \mathrm{T}_{\mathrm{i}} \mathrm{X} \forall \mathrm{i}$, corresponding to each $\mathrm{x}_{\mathrm{n}}$ there exists $\mathrm{y}_{\mathrm{n}}$ in $\mathrm{X}$ such that $\mathrm{A}_{\mathrm{i}} \mathrm{x}_{\mathrm{n}}=\mathrm{T}_{\mathrm{i}} \mathrm{y}_{\mathrm{n}} \forall \mathrm{i}$ and

$\mathrm{A}_{\mathrm{i}} \mathrm{x}_{\mathrm{n}}=\mathrm{T}_{\mathrm{i}} \mathrm{y}_{\mathrm{n}} \rightarrow$ t.By using (ii) and we have $\lim _{n \rightarrow \infty} A_{i} y_{n}=\mathrm{t} \forall \mathrm{i}$.

Thus we get sequences $\left\{\mathrm{x}_{\mathrm{n}}\right\}$ and $\left\{\mathrm{y}_{\mathrm{n}}\right\}$ in $\mathrm{X}$ such that $\mathrm{A}_{\mathrm{i}} \mathrm{x}_{\mathrm{n}} \rightarrow \mathrm{t}, \mathrm{S}_{\mathrm{i}} \mathrm{x}_{\mathrm{n}}-\rightarrow \mathrm{t}, \mathrm{T}_{\mathrm{i}} \mathrm{y}_{\mathrm{n}} \rightarrow \mathrm{t}$ and $\mathrm{A}_{\mathrm{i}} \mathrm{y}_{\mathrm{n}} \rightarrow \mathrm{t} \forall \mathrm{i}$.

where $\mathrm{T}_{\mathrm{i}} \mathrm{y}_{\mathrm{n}}=\mathrm{A}_{\mathrm{i}} \mathrm{x}_{\mathrm{n}} \forall \mathrm{i}$.

Now, suppose that $\mathrm{S}_{\mathrm{i}} \forall \mathrm{i}$, the range of $\mathrm{S}_{\mathrm{i}} \forall \mathrm{i}$ is a complete subspace of X.Then, Since $\lim _{n \rightarrow \infty} S_{i} x_{n}=\mathrm{t} \forall \mathrm{i}$, there exists a point $\mathrm{u}$ in $\mathrm{X}$ such that $\mathrm{t}=\mathrm{S}_{\mathrm{i}} \mathrm{u} \forall \mathrm{i}$

Therefore, $\lim _{n \rightarrow \infty} A_{i} x_{n}=\lim _{n \rightarrow \infty} B_{i} y_{n}=\lim _{n \rightarrow \infty} T_{i} y_{n}=\lim _{n \rightarrow \infty} S_{i} x_{n}=\mathrm{S}_{\mathrm{i}} \mathrm{u} \forall \mathrm{i}$

$\mathrm{d}\left(\mathrm{A}_{\mathrm{i}} \mathrm{u}, \mathrm{B}_{\mathrm{i}} \mathrm{y}_{\mathrm{n}}\right)<\mathrm{M}_{\mathrm{ii}}(\mathrm{x}, \mathrm{y})=\max \left\{\mathrm{d}\left(\mathrm{S}_{\mathrm{i}} \mathrm{u}, \mathrm{T}_{\mathrm{i}} \mathrm{y}_{\mathrm{n}}\right), \mathrm{d}\left(\mathrm{A}_{\mathrm{i}} \mathrm{u}, \mathrm{S}_{\mathrm{i}} \mathrm{u}\right), \mathrm{d}\left(\mathrm{B}_{\mathrm{i}} \mathrm{y}_{\mathrm{n}}, \mathrm{T}_{\mathrm{i}} \mathrm{y}_{\mathrm{n}}\right),\left[\mathrm{d}\left(\mathrm{S}_{\mathrm{i}} \mathrm{u}, \mathrm{B}_{\mathrm{i}} \mathrm{y}_{\mathrm{n}}\right)+\mathrm{d}\left(\mathrm{A}_{\mathrm{i}} \mathrm{u}, \mathrm{T}_{\mathrm{i}} \mathrm{y}_{\mathrm{n}}\right)\right] / 2\right\} \forall \mathrm{i}$.

$=\operatorname{Max}\left\{\mathrm{d}\left(\mathrm{A}_{\mathrm{i}} \mathrm{u}, \mathrm{B}_{\mathrm{i}} \mathrm{y}_{\mathrm{n}}\right), 0\right\}=\mathrm{d}\left(\mathrm{A}_{\mathrm{i}} \mathrm{u}, \mathrm{B}_{\mathrm{i}} \mathrm{y}_{\mathrm{n}}\right) \forall \mathrm{i}$.

Therefore, $\mathrm{d}\left(\mathrm{A}_{\mathrm{i}} \mathrm{u}, \mathrm{B}_{\mathrm{i}} \mathrm{y}_{\mathrm{n}}\right)<\mathrm{d}\left(\mathrm{A}_{\mathrm{i}} \mathrm{u}, \mathrm{B}_{\mathrm{i}} \mathrm{y}_{\mathrm{n}}\right) \forall \mathrm{i}$. Which is a contradiction.

Hence $\mathrm{A}_{\mathrm{i}} \mathrm{u}=\mathrm{S}_{\mathrm{i}} \mathrm{u} \forall{ }_{\mathrm{i}}$.

Since $A_{i} X \subset T_{i} X \forall i$, there exists $w$ in $X$ such that $A_{i} u=T_{i} W \forall$ i. If $A_{i} u \neq B_{i} w$ for all i,uing (ii)

We obtain d $\left(\mathrm{A}_{\mathrm{i}} \mathrm{u}, \mathrm{B}_{\mathrm{i}} \mathrm{w}\right)<\mathrm{M}_{\mathrm{ii}}(\mathrm{u}, \mathrm{w})=\operatorname{Max}\left\{\mathrm{d}\left(\mathrm{S}_{\mathrm{i}} \mathrm{u}, \mathrm{T}_{\mathrm{i}} \mathrm{w}\right), \mathrm{d}\left(\mathrm{A}_{\mathrm{i}} \mathrm{u}, \mathrm{S}_{\mathrm{i}} \mathrm{u}\right), \mathrm{d}\left(\mathrm{B}_{\mathrm{i}} \mathrm{w}, \mathrm{T}_{\mathrm{i}} \mathrm{w}\right),\left[\mathrm{d}\left(\mathrm{S}_{\mathrm{i}} \mathrm{u}, \mathrm{B}_{\mathrm{i}} \mathrm{w}\right)+\mathrm{d}\left(\mathrm{A}_{\mathrm{i}} \mathrm{u}, \mathrm{T}_{\mathrm{i}} \mathrm{w}\right)\right] / 2\right\} . \forall \mathrm{i}$.

$=\max \left\{\mathrm{d}\left(\mathrm{B}_{\mathrm{i}} \mathrm{w}, \mathrm{A}_{\mathrm{i}} \mathrm{w}\right),\left[\mathrm{d}\left(\mathrm{A}_{\mathrm{i}} \mathrm{u}, \mathrm{B}_{\mathrm{i}} \mathrm{w}\right)+0\right] / 2\right\}=\mathrm{d}\left(\mathrm{A}_{\mathrm{i}} \mathrm{u}, \mathrm{B}_{\mathrm{i}} \mathrm{w}\right)$.

$\mathrm{d}\left(\mathrm{A}_{\mathrm{i}} \mathrm{u}, \mathrm{B}_{\mathrm{i}} \mathrm{w}\right)<\mathrm{d}\left(\mathrm{A}_{\mathrm{i}} \mathrm{u}, \mathrm{B}_{\mathrm{i}} \mathrm{w}\right) \forall \mathrm{i}$. Which is a contradiction

Hence, $\mathrm{S}_{\mathrm{i}} \mathrm{u}=\mathrm{A}_{\mathrm{i}} \mathrm{u}=\mathrm{T}_{\mathrm{i}} \mathrm{W}=\mathrm{B}_{\mathrm{i}} \mathrm{W} \quad \forall \mathrm{i}$.

Next let us assume that $T_{i} X \forall i$ is a complete subspace of $X$.

Then since $\lim _{n \rightarrow \infty} T_{i} y_{n}=\mathrm{t} \forall \mathrm{i}$ there exists a point $\mathrm{w}$ in $\mathrm{X}$ such that $\mathrm{t}=\mathrm{T}_{\mathrm{i}} \mathrm{W} \forall \mathrm{i}$

If $\mathrm{B}_{\mathrm{i}} \mathrm{W} \neq \mathrm{T}_{\mathrm{i}} \mathrm{W}$ using (ii) for sufficiently large values of $\mathrm{n}$, 
We get $d\left(\mathrm{~A}_{\mathrm{i}} \mathrm{X}_{\mathrm{n}}, \mathrm{B}_{\mathrm{i}} \mathrm{W}\right) \leq \mathrm{M}_{\mathrm{ii}}(\mathrm{x}, \mathrm{y})=\max \left\{\mathrm{d}\left(\mathrm{S}_{\mathrm{iX}}, \mathrm{T}_{\mathrm{i}} \mathrm{W}\right), \mathrm{d}\left(\mathrm{A}_{\mathrm{iX}}, \mathrm{S}_{\mathrm{iX}_{\mathrm{n}}}\right), \mathrm{d}\left(\mathrm{B}_{\mathrm{i}} \mathrm{w}, \mathrm{T}_{\mathrm{i}} \mathrm{W}\right),\left[\mathrm{d}\left(\mathrm{S}_{\mathrm{iX}_{\mathrm{n}}}, \mathrm{B}_{\mathrm{i}} \mathrm{W}\right)+\mathrm{d}\left(\mathrm{A}_{\mathrm{iX}}, \mathrm{T}_{\mathrm{i}} \mathrm{w}\right)\right] / 2\right\}$ On letting $\mathrm{n} \rightarrow \infty$, we have $\mathrm{d}\left(\mathrm{T}_{\mathrm{i}} \mathrm{w}, \mathrm{B}_{\mathrm{i}} \mathrm{w}\right)<\mathrm{d}\left(\mathrm{T}_{\mathrm{i}} \mathrm{w}, \mathrm{B}_{\mathrm{i}} \mathrm{w}\right)$ Which is a contradiction.

Hence $\mathrm{T}_{\mathrm{i}} \mathrm{w}=\mathrm{B}_{\mathrm{i}} \mathrm{W} \forall \mathrm{i}$.

Since $\mathrm{B}_{\mathrm{i}} \mathrm{X} \subset \mathrm{S}_{\mathrm{i}} \mathrm{X} \forall \mathrm{i}$, there exists $\mathrm{u}$ in $\mathrm{X}$ such that $\mathrm{T}_{\mathrm{i}} \mathrm{W}=\mathrm{A}_{\mathrm{i}} \mathrm{W}=\mathrm{S}_{\mathrm{i}} \mathrm{u} \forall \mathrm{i}$

using (ii) we get $\mathrm{T}_{\mathrm{i}} \mathrm{W}=\mathrm{B}_{\mathrm{i}} \mathrm{W}=\mathrm{S}_{\mathrm{i}} \mathrm{u}=\mathrm{A}_{\mathrm{i}} \mathrm{u} \forall \mathrm{i}$

Again using (ii) we get $\mathrm{S}_{\mathrm{i}} \mathrm{u}=\mathrm{A}_{\mathrm{i}} \mathrm{u}=\mathrm{T}_{\mathrm{i}} \mathrm{W}=\mathrm{B}_{\mathrm{i}} \mathrm{W} \forall \mathrm{i}$

Thus irrespective of whether $\mathrm{S}_{\mathrm{i}} \mathrm{X} \forall \mathrm{i}$ is assumed complete or $\mathrm{T}_{\mathrm{i}} \mathrm{X} \forall \mathrm{i}$ is assumed to be so.

we get $\mathrm{u} u, \mathrm{w}$ in $\mathrm{X}$ such that $\mathrm{A}_{\mathrm{i}} \mathrm{u}=\mathrm{S}_{\mathrm{i}} \mathrm{u}=\mathrm{T}_{\mathrm{i}} \mathrm{W}=\mathrm{B}_{\mathrm{i}} \mathrm{W} \forall \mathrm{i}$

Point wise R-weak commutativity of $A_{i}$ and $S_{i} \forall i$ implies that there exists $R_{1}>0$ such that

$\mathrm{d}\left(\mathrm{A}_{\mathrm{i}} \mathrm{S}_{\mathrm{i}} \mathrm{u}, \mathrm{S}_{\mathrm{i}} \mathrm{A}_{\mathrm{i}} \mathrm{u}\right) \leq \mathrm{R}_{1} \mathrm{~d}\left(\mathrm{~A}_{\mathrm{i}} \mathrm{u}, \mathrm{S}_{\mathrm{i}} \mathrm{u}\right)=0$

That is $\mathrm{A}_{\mathrm{i}} \mathrm{S}_{\mathrm{i}} \mathrm{u}=\mathrm{S}_{\mathrm{i}} \mathrm{A}_{\mathrm{i}} \mathrm{u} \quad \forall \mathrm{i}$ and $\mathrm{A}_{\mathrm{i}} \mathrm{A}_{\mathrm{i}} \mathrm{u}=\mathrm{A}_{\mathrm{i}} \mathrm{S}_{\mathrm{i}} \mathrm{u}=\mathrm{S}_{\mathrm{i}} \mathrm{A}_{\mathrm{i}} \mathrm{u}=\mathrm{S}_{\mathrm{i}} \mathrm{S}_{\mathrm{i}} \mathrm{u} \quad \forall \mathrm{i}$

Similarly, for every $\mathrm{i}$, there exists $\mathrm{R}_{\mathrm{i}}>0$ such that $\mathrm{d}\left(\mathrm{B}_{\mathrm{i}} \mathrm{T}_{\mathrm{i}} \mathrm{w}, \mathrm{T}_{\mathrm{i}} \mathrm{B}_{\mathrm{i}} \mathrm{w}\right) \leq \mathrm{R}_{\mathrm{i}} \mathrm{d}\left(\mathrm{B}_{\mathrm{i}} \mathrm{w}, \mathrm{T}_{\mathrm{i}} \mathrm{w}\right)=0$, that is

$\mathrm{B}_{\mathrm{i}} \mathrm{w} \mathrm{T}_{\mathrm{i}} \mathrm{W}=\mathrm{T}_{\mathrm{i}} \mathrm{W} \mathrm{B}_{\mathrm{i}} \mathrm{W} \forall \mathrm{i}$ and $\mathrm{B}_{\mathrm{i}} \mathrm{wB}_{\mathrm{i}} \mathrm{W}=\mathrm{B}_{\mathrm{i}} \mathrm{WT} \mathrm{T}_{\mathrm{i}} \mathrm{W}=\mathrm{T}_{\mathrm{i}} \mathrm{W} \mathrm{B}_{\mathrm{i}} \mathrm{W}=\mathrm{T}_{\mathrm{i}} \mathrm{WT} \mathrm{T}_{\mathrm{i}} \mathrm{W} \forall \mathrm{i}$

If $\mathrm{A}_{\mathrm{i}} \mathrm{A}_{\mathrm{i}} \mathrm{u} \neq \mathrm{A}_{\mathrm{i}} \mathrm{u} \forall \mathrm{i}$, using (ii) weget $\mathrm{d}\left(\mathrm{A}_{\mathrm{i}} \mathrm{A}_{\mathrm{i}} \mathrm{u}, \mathrm{A}_{\mathrm{i}} \mathrm{u}\right)=\mathrm{d}\left(\mathrm{A}_{\mathrm{i}} \mathrm{A}_{\mathrm{i}} \mathrm{u}, \mathrm{B}_{\mathrm{i}} \mathrm{w}\right)<\mathrm{M}_{\mathrm{ii}}\left(\mathrm{A}_{\mathrm{i}} \mathrm{u}, \mathrm{w}\right)=\mathrm{d}\left(\mathrm{A}_{\mathrm{i}} \mathrm{A}_{\mathrm{i}} \mathrm{u}, \mathrm{B}_{\mathrm{i}} \mathrm{w}\right) \forall \mathrm{i}$

Which is a contradiction. Hence $A_{i} u=A_{i} A_{i} u=S_{i} A_{i} u{ }_{i}$ and $A_{i} u$ is a common fixed point of $A_{i}$ and $S_{i} \forall i$. Similarly, if $\mathrm{B}_{\mathrm{i}} \mathrm{B}_{\mathrm{i}} \mathrm{W} \neq \mathrm{B}_{\mathrm{i}} \mathrm{W} \forall \mathrm{i}$ using (ii) we have $\mathrm{d}\left(\mathrm{B}_{\mathrm{i}} \mathrm{w} \mathrm{B}_{\mathrm{i}} \mathrm{B}_{\mathrm{i}} \mathrm{w}\right)=\mathrm{d}\left(\mathrm{A}_{\mathrm{i}} \mathrm{u}, \mathrm{B}_{\mathrm{i}} \mathrm{B}_{\mathrm{i}} \mathrm{w}\right)<\mathrm{M}_{\mathrm{ii}}\left(\mathrm{u}, \mathrm{B}_{\mathrm{i}} \mathrm{w}\right)=\mathrm{d}\left(\mathrm{A}_{\mathrm{i}} \mathrm{u}, \mathrm{B}_{\mathrm{i}} \mathrm{B}_{\mathrm{i}} \mathrm{w}\right), \forall \mathrm{i}$ which is a contradiction.

Hence $\mathrm{B}_{\mathrm{i}} \mathrm{W}=\mathrm{B}_{\mathrm{i}} \mathrm{B}_{\mathrm{i}} \mathrm{W}=\mathrm{T}_{\mathrm{i}} \mathrm{B}_{\mathrm{i}} \mathrm{W} \forall \mathrm{i}$ that is $\mathrm{B}_{\mathrm{i}} \mathrm{W}=\mathrm{A}_{\mathrm{i}} \mathrm{u}$ is a common fixed point of $\mathrm{T}_{\mathrm{i}}$ and $\mathrm{B}_{\mathrm{i}} \forall \mathrm{i}$

Uniqueness. Suppose $\mathrm{u}, \mathrm{v}$ are fixed point of $\mathrm{A}_{\mathrm{i}}, \mathrm{B}_{\mathrm{i}}, \mathrm{T}_{\mathrm{i}}$ and $\mathrm{S}_{\mathrm{i}} \forall_{\mathrm{i}}$

Then $\mathrm{A}_{\mathrm{i}} \mathrm{u}=\mathrm{S}_{\mathrm{i}} \mathrm{u}=\mathrm{B}_{\mathrm{i}} \mathrm{u}=\mathrm{T}_{\mathrm{i}} \mathrm{u}=\mathrm{u} \forall \mathrm{i}$ and

$\mathrm{A}_{\mathrm{i}} \mathrm{v}=\mathrm{S}_{\mathrm{i}} \mathrm{v}=\mathrm{B}_{\mathrm{i}} \mathrm{v}=\mathrm{T}_{\mathrm{i}} \mathrm{v}=\mathrm{v} \forall \mathrm{i}$

$\mathrm{d}(\mathrm{u}, \mathrm{v})=\mathrm{d}\left(\mathrm{A}_{\mathrm{i}} \mathrm{u}, \mathrm{B}_{\mathrm{i}} \mathrm{v}\right)<\max \left\{\mathrm{d}\left(\mathrm{S}_{\mathrm{i}} \mathrm{u}, \mathrm{T}_{\mathrm{i}} \mathrm{v}\right), \mathrm{d}\left(\mathrm{A}_{\mathrm{i}} \mathrm{u}, \mathrm{S}_{\mathrm{i}} \mathrm{u}\right), \mathrm{d}\left(\mathrm{B}_{\mathrm{i}} \mathrm{v}, \mathrm{T}_{\mathrm{i}} \mathrm{v}\right),\left[\mathrm{d}\left(\mathrm{S}_{\mathrm{i}} \mathrm{u}, \mathrm{B}_{\mathrm{i}} \mathrm{v}\right)+\mathrm{d}\left(\mathrm{A}_{\mathrm{i}} \mathrm{u}, \mathrm{T}_{\mathrm{i}} \mathrm{v}\right)\right] / 2\right\}$

$=\max \{\mathrm{d}(\mathrm{u}, \mathrm{v}), 0,0,[\mathrm{~d}(\mathrm{u}, \mathrm{v})+\mathrm{d}(\mathrm{u}, \mathrm{v})] / 2\}=\max \{\mathrm{d}(\mathrm{u}, \mathrm{v}), \mathrm{d}(\mathrm{u}, \mathrm{v})\}=\mathrm{d}(\mathrm{u}, \mathrm{v})$

$=><=$ when $\mathrm{u} \neq \mathrm{v}$.

Therefore $\mathrm{u}=\mathrm{v}$.

The proof is similar when $\mathrm{B}_{\mathrm{i}} \mathrm{X}$ is assumed complete for some $\mathrm{i}$.

Since, $\mathrm{A}_{\mathrm{i}} \mathrm{X} \subset \mathrm{T}_{\mathrm{i}} \mathrm{X}$ and $\mathrm{B}_{\mathrm{i}} \mathrm{X} \subset \mathrm{S}_{\mathrm{i}} \mathrm{X} \forall \mathrm{i}$.

Therefore proof is complete.

Theorem 3.2. Let $\left\{\mathrm{A}_{\mathrm{i}}\right\},\left\{\mathrm{B}_{\mathrm{i}}\right\},\left\{\mathrm{S}_{\mathrm{i}}\right\},\left\{\mathrm{T}_{\mathrm{i}}\right\} \forall \mathrm{i}=1,2,3, \ldots$ be self-mappings of a metric space $(\mathrm{X}, \mathrm{d})$ such that $\mathrm{A}_{\mathrm{i}} \mathrm{X} \subset$ $\mathrm{T}_{\mathrm{i}} \mathrm{X}, \mathrm{B}_{\mathrm{i}} \mathrm{X} \subset \mathrm{S}_{\mathrm{i}} \mathrm{X} \forall \mathrm{i}$ and (i) Pairs $\left(\mathrm{A}_{\mathrm{i}}, \mathrm{S}_{\mathrm{i}}\right)$ and $\left(\mathrm{B}_{\mathrm{i}}, \mathrm{T}_{\mathrm{i}}\right) \forall \mathrm{i}$ are Point wise R-weakly commuting with atleast one non pair compatible, one non Compatible.

(ii) $\mathrm{d}\left(\mathrm{A}_{\mathrm{i}} \mathrm{x}, \mathrm{B}_{\mathrm{i}} \mathrm{y}\right)<\mathrm{M}_{\mathrm{ii}}(\mathrm{x}, \mathrm{y})=\max \left\{\mathrm{d}\left(\mathrm{S}_{\mathrm{i}} \mathrm{x}, \mathrm{T}_{\mathrm{i}} \mathrm{y}\right), \mathrm{d}\left(\mathrm{A}_{\mathrm{i}} \mathrm{x}, \mathrm{S}_{\mathrm{i}} \mathrm{x}\right), \mathrm{d}\left(\mathrm{B}_{\mathrm{i}} \mathrm{y}, \mathrm{T}_{\mathrm{i}} \mathrm{y}\right), \mathrm{d}\left(\mathrm{S}_{\mathrm{i}} \mathrm{x}, \mathrm{B}_{\mathrm{i}} \mathrm{y}\right), \mathrm{d}\left(\mathrm{A}_{\mathrm{i}} \mathrm{x}, \mathrm{T}_{\mathrm{i}} \mathrm{y}\right)\right\} \forall$ i whenever $\mathrm{M}_{\mathrm{ii}}(\mathrm{x}, \mathrm{y})>0$. If one of the mappings in the Compatible pair is continuous then all the $\mathrm{A}_{\mathrm{i}}, \mathrm{B}_{\mathrm{i}}, \mathrm{S}_{\mathrm{i}}$ andT $\mathrm{T}_{\mathrm{i}} \forall \mathrm{i}$ have a unique common fixed point.

Proof. Let $\mathrm{B}_{\mathrm{i}}$ and $\mathrm{T}_{\mathrm{i}} \forall \mathrm{i}$ be a non compatible mappings and $\mathrm{A}_{\mathrm{i}}$ and $\mathrm{S}_{\mathrm{i}} \forall \mathrm{i}$ be continuous compatible mappings. Then non compatible of $\mathrm{B}_{\mathrm{i}}$ and $\mathrm{T}_{\mathrm{i}} \forall \mathrm{i}$ implies that there exists some sequence $\left\{\mathrm{x}_{\mathrm{n}}\right\}$ in $\mathrm{X}$ such that $\lim _{n \rightarrow \infty} B_{i} x_{n}=\lim _{n \rightarrow \infty} T_{i} x_{n}=\mathrm{t} \forall \mathrm{i}$ for some $\mathrm{t}$ in $\mathrm{X}$

While $\lim _{n \rightarrow \infty} d\left(B_{i} T_{i} x_{n}, T_{i} B_{i} x_{n}\right) \forall \mathrm{i}$ is either non zero or nonexistent. Since $\mathrm{B}_{\mathrm{i}} \mathrm{X} \subset \mathrm{S}_{\mathrm{i}} \mathrm{X} \forall \mathrm{i}$

Corresponding to each $\mathrm{x}_{\mathrm{n}}$ there exists a $\mathrm{y}_{\mathrm{n}}$ in $\mathrm{X}$ such that $B_{i} x_{n}=\mathrm{S}_{\mathrm{i}} \mathrm{y}_{\mathrm{n}} \forall \mathrm{i}$.

Thus $B_{i} x_{n} \rightarrow \mathrm{t}, T_{i} x_{n} \rightarrow \mathrm{t}$ and $\mathrm{S}_{\mathrm{i}} \mathrm{y}_{\mathrm{n}} \rightarrow \mathrm{t} \forall \mathrm{i}$.

We claim that $\mathrm{A}_{\mathrm{i}} \mathrm{y}_{\mathrm{n}} \rightarrow \mathrm{t} \forall \mathrm{i}$. If not, then there exists a subsequence $\left\{\mathrm{A}_{\mathrm{i}} \mathrm{y}_{\mathrm{m}}\right\}$ of $\left\{\mathrm{A}_{\mathrm{i}} \mathrm{y}_{\mathrm{n}}\right\} \forall \mathrm{i}$

a number $\mathrm{r}>0$ and a positive integer $\mathrm{M}$ such that for each $\mathrm{m} \geq \mathrm{M}$, we have $\mathrm{d}\left(\mathrm{A}_{\mathrm{i}} \mathrm{y}_{\mathrm{m}}, \mathrm{t}\right) \geq \mathrm{r}, \mathrm{d}\left(\mathrm{A}_{\mathrm{i}} \mathrm{y}_{\mathrm{m}}, B_{i} x_{m}\right) \geq \mathrm{r} \forall \mathrm{i}$ and $\mathrm{d}\left(\mathrm{A}_{\mathrm{i}} \mathrm{y}_{\mathrm{m}}, B_{i} x_{m}\right)<\max \left\{\mathrm{d}\left(\mathrm{S}_{\mathrm{i}} \mathrm{y}_{\mathrm{m}}, \mathrm{T}_{\mathrm{i}} \mathrm{x}_{\mathrm{m}}\right), \mathrm{d}\left(\mathrm{A}_{\mathrm{i}} \mathrm{y}_{\mathrm{m}}, \mathrm{S}_{\mathrm{i}} \mathrm{y}_{\mathrm{m}}\right), \mathrm{d}\left(\mathrm{B}_{\mathrm{i}} \mathrm{x}_{\mathrm{m}}, \mathrm{T}_{\mathrm{i}} \mathrm{x}_{\mathrm{m}}\right), \mathrm{d}\left(\mathrm{S}_{\mathrm{i}} \mathrm{y}_{\mathrm{m}}, \mathrm{B}_{\mathrm{i}} \mathrm{x}_{\mathrm{m}}\right), \mathrm{d}\left(\mathrm{A}_{\mathrm{i}} \mathrm{y}_{\mathrm{m}}, \mathrm{T}_{\mathrm{i}} \mathrm{x}_{\mathrm{m}}\right)\right\} \forall \mathrm{i}$

$=\max \left\{\mathrm{d}\left(\mathrm{A}_{\mathrm{i}} \mathrm{y}_{\mathrm{m}}, \mathrm{B}_{\mathrm{i}} \mathrm{x}_{\mathrm{m}}\right), \mathrm{d}\left(\mathrm{A}_{\mathrm{i}} \mathrm{y}_{\mathrm{m}}, \mathrm{B}_{\mathrm{i}} \mathrm{x}_{\mathrm{m}}\right)\right\}=\mathrm{d}\left(\mathrm{A}_{\mathrm{i}} \mathrm{y}_{\mathrm{m}}, \mathrm{B}_{\mathrm{i}} \mathrm{x}_{\mathrm{m}}\right) \forall \mathrm{i}$. which is a contradiction.

Hence $\lim _{n \rightarrow \infty} A_{i} y_{n}=t, \lim _{n \rightarrow \infty} S_{i} y_{n}=\mathrm{t}, \lim _{n \rightarrow \infty} B_{i} x_{n}=t$, and $\lim _{n \rightarrow \infty} T_{i} x_{n}=\mathrm{t} \forall \mathrm{i}$,

Where $\mathrm{S}_{\mathrm{i}} \mathrm{y}_{\mathrm{n}}=\mathrm{B}_{\mathrm{i}} \mathrm{x}_{\mathrm{n}} \forall \mathrm{i}$. Since, $\mathrm{A}_{\mathrm{i}}$ and $\mathrm{S}_{\mathrm{i}} \forall \mathrm{i}$ are continuous, we get $\lim _{n \rightarrow \infty} S_{i} A_{i} y_{n}=\mathrm{S}_{\mathrm{i}} \mathrm{t} \forall \mathrm{i}$

and $\lim _{n \rightarrow \infty} A_{i} S_{i} y_{n}=\mathrm{A}_{\mathrm{i}} \mathrm{t} \forall \mathrm{i}$ compatibility of $\mathrm{A}_{\mathrm{i}}$ and $\mathrm{S}_{\mathrm{i}} \forall \mathrm{i}$ implies that $\lim _{n \rightarrow \infty} d\left(A_{i} S_{i} y_{n}, S_{i} A_{i} y_{n}\right)=0 \forall \mathrm{i}$. That is, $\mathrm{d}\left(\mathrm{A}_{\mathrm{i}} \mathrm{t}, \mathrm{S}_{\mathrm{i}} \mathrm{t}\right)=0 \forall \mathrm{i}$.

Thus $\mathrm{A}_{\mathrm{i}} \mathrm{t}=\mathrm{S}_{\mathrm{i}} \mathrm{t}, \forall \mathrm{i}$ Since $\mathrm{A}_{\mathrm{i}} \mathrm{X} \subset \mathrm{T}_{\mathrm{i}} \mathrm{X} \forall \mathrm{i}$, there exists some point $\mathrm{w}$ in $\mathrm{X}$ such that $\mathrm{A}_{\mathrm{i}} \mathrm{t}=\mathrm{T}_{\mathrm{i}} \mathrm{W} \forall \mathrm{i}$

Now, if $\mathrm{T}_{\mathrm{i}} \mathrm{w} \neq \mathrm{B}_{\mathrm{i}} \mathrm{w} \forall \mathrm{i}$. 
$\mathrm{d}\left(\mathrm{A}_{\mathrm{i}} \mathrm{t}, \mathrm{B}_{\mathrm{i}} \mathrm{w}\right)<\max \left\{\mathrm{d}\left(\mathrm{S}_{\mathrm{i}} \mathrm{t}, \mathrm{T}_{\mathrm{i}} \mathrm{w}\right), \mathrm{d}\left(\mathrm{A}_{\mathrm{i}} \mathrm{t}, \mathrm{S}_{\mathrm{i}} \mathrm{t}\right), \mathrm{d}\left(\mathrm{B}_{\mathrm{i}} \mathrm{w}, \mathrm{T}_{\mathrm{i}} \mathrm{w}\right), \mathrm{d}\left(\mathrm{S}_{\mathrm{i}} \mathrm{t}, \mathrm{B}_{\mathrm{i}} \mathrm{w}\right), \mathrm{d}\left(\mathrm{A}_{\mathrm{i}} \mathrm{t}, \mathrm{T}_{\mathrm{i}} \mathrm{w}\right)\right\} \forall{ }_{\mathrm{i}}$

$\mathrm{d}\left(\mathrm{A}_{\mathrm{i}} \mathrm{t}, \mathrm{B}_{\mathrm{i}} \mathrm{w}\right)<\max \left\{\mathrm{d}\left(\mathrm{B}_{\mathrm{i}} \mathrm{w}, \mathrm{A}_{\mathrm{i}} \mathrm{t}\right), \mathrm{d}\left(\mathrm{A}_{\mathrm{i}} \mathrm{t}, \mathrm{B}_{\mathrm{i}} \mathrm{w}\right)\right\}=\mathrm{d}\left(\mathrm{B}_{\mathrm{i}} \mathrm{w}, \mathrm{A}_{\mathrm{i}} \mathrm{t}\right)$

Therefore, $\mathrm{d}\left(\mathrm{A}_{\mathrm{i}} \mathrm{t}, \mathrm{B}_{\mathrm{i}} \mathrm{w}\right)<\mathrm{d}\left(\mathrm{A}_{\mathrm{i}} \mathrm{t}, \mathrm{B}_{\mathrm{i}} \mathrm{W}\right) \forall \mathrm{i}$. Which is a contradiction.

Hence $\mathrm{B}_{\mathrm{i}} \mathrm{W}=\mathrm{T}_{\mathrm{i}} \mathrm{W} \forall \mathrm{i}$ and $\mathrm{S}_{\mathrm{i}} \mathrm{t}=\mathrm{A}_{\mathrm{i}} \mathrm{t}=\mathrm{T}_{\mathrm{i}} \mathrm{W}=\mathrm{B}_{\mathrm{i}} \mathrm{W} \forall \mathrm{i}$

Point wise R-weak commutativity of $\mathrm{B}_{\mathrm{i}}$ and $\mathrm{T}_{\mathrm{i}} \forall \mathrm{i}$ implies that there exists $\mathrm{R}>0$ such that

$\mathrm{d}\left(\mathrm{B}_{\mathrm{i}} \mathrm{T}_{\mathrm{i}} \mathrm{w}, \mathrm{T}_{\mathrm{i}} \mathrm{B}_{\mathrm{i}} \mathrm{w}\right) \leq \mathrm{Rd}\left(\mathrm{B}_{\mathrm{i}} \mathrm{w}, \mathrm{T}_{\mathrm{i}} \mathrm{w}\right)=0 \forall \mathrm{i}$.

That is, $\mathrm{B}_{\mathrm{i}} \mathrm{T}_{\mathrm{i}} \mathrm{W}=\mathrm{T}_{\mathrm{i}} \mathrm{B}_{\mathrm{i}} \mathrm{W} \forall \mathrm{i}$. More over $\mathrm{B}_{\mathrm{i}} \mathrm{B}_{\mathrm{i}} \mathrm{W}=\mathrm{B}_{\mathrm{i}} \mathrm{T}_{\mathrm{i}} \mathrm{W}=\mathrm{T}_{\mathrm{i}} \mathrm{B}_{\mathrm{i}} \mathrm{W}=\mathrm{T}_{\mathrm{i}} \mathrm{T}_{\mathrm{i}} \mathrm{W} \forall \mathrm{i}$

Similarly, compatibility of $A_{i}$ and $S_{i} \forall i$ implies that $A_{i} S_{i} t=S_{i} A_{i} t$ and

$\mathrm{A}_{\mathrm{i}} \mathrm{A}_{\mathrm{i}} \mathrm{t}=\mathrm{S}_{\mathrm{i}} \mathrm{A}_{\mathrm{i}} \mathrm{t}=\mathrm{S}_{\mathrm{i}} \mathrm{S}_{\mathrm{i}} \mathrm{t} \forall$ i. Now if $\mathrm{A}_{\mathrm{i}} \mathrm{t} \neq \mathrm{A}_{\mathrm{i}} \mathrm{A}_{\mathrm{i}} \mathrm{t} \forall \mathrm{i}$, using (ii) we get $\mathrm{d}\left(\mathrm{A}_{\mathrm{i}} \mathrm{t}, \mathrm{A}_{\mathrm{i}} \mathrm{A}_{\mathrm{i}} \mathrm{t}\right)=\mathrm{d}\left(\mathrm{A}_{\mathrm{i}} \mathrm{A}_{\mathrm{i}} \mathrm{t}, \mathrm{B}_{\mathrm{i}} \mathrm{w}\right)$

$<\mathrm{M}_{\mathrm{ii}}\left(\mathrm{A}_{\mathrm{i}} \mathrm{t}, \mathrm{w}\right)=\mathrm{d}\left(\mathrm{A}_{\mathrm{i}} \mathrm{A}_{\mathrm{i}} \mathrm{t}, \mathrm{B}_{\mathrm{i}} \mathrm{w}\right) \forall \mathrm{i}$. Which is a contradiction.

Hence, $\mathrm{A}_{\mathrm{i}} \mathrm{t}=\mathrm{A}_{\mathrm{i}} \mathrm{A}_{\mathrm{i}} \mathrm{t}=\mathrm{S}_{\mathrm{i}} \mathrm{A}_{\mathrm{i}} \mathrm{t} \quad \forall \mathrm{i}$ and $\mathrm{A}_{\mathrm{i}} \mathrm{t} \forall \mathrm{i}$ is a common fixed point of $\mathrm{A}_{\mathrm{i}}$ and $\mathrm{S}_{\mathrm{i}} \forall \mathrm{i}$

Similarly, $\mathrm{B}_{\mathrm{i}} \mathrm{w}\left(=\mathrm{A}_{\mathrm{i}} \mathrm{t}\right) \forall \mathrm{i}$ is a common fixed point of $\mathrm{B}_{\mathrm{i}}$ and $\mathrm{T}_{\mathrm{i}} \forall \mathrm{i}$.

Uniqueness. Suppose $\mathrm{u}, \mathrm{v}$ are fixed points of $\mathrm{A}_{\mathrm{i}}, \mathrm{B}_{\mathrm{i}}, \mathrm{S}_{\mathrm{i}}$ and $\mathrm{T}_{\mathrm{i}} \forall \mathrm{i}$.

Then $\mathrm{A}_{\mathrm{i}} \mathrm{u}=\mathrm{S}_{\mathrm{i}} \mathrm{u}=\mathrm{B}_{\mathrm{i}} \mathrm{u}=\mathrm{T}_{\mathrm{i}} \mathrm{u}=\mathrm{u} \forall \mathrm{i}$ and

$\mathrm{A}_{\mathrm{i}} \mathrm{v}=\mathrm{S}_{\mathrm{i}} \mathrm{v}=\mathrm{B}_{\mathrm{i}} \mathrm{v}=\mathrm{T}_{\mathrm{i}} \mathrm{v}=\mathrm{v} \forall \mathrm{i} \mathrm{d}(\mathrm{u}, \mathrm{v})=\mathrm{d}\left(\mathrm{A}_{\mathrm{i}} \mathrm{u}, \mathrm{B}_{\mathrm{i}} \mathrm{v}\right)<\max \left\{\mathrm{d}\left(\mathrm{S}_{\mathrm{i}} \mathrm{u}, \mathrm{T}_{\mathrm{i}} \mathrm{v}\right), \mathrm{d}\left(\mathrm{A}_{\mathrm{i}} \mathrm{u}, \mathrm{S}_{\mathrm{i}} \mathrm{u}\right), \mathrm{d}\left(\mathrm{B}_{\mathrm{i}} \mathrm{v}, \mathrm{T}_{\mathrm{i}} \mathrm{v}\right), \mathrm{d}\left(\mathrm{S}_{\mathrm{i}} \mathrm{u}, \mathrm{B}_{\mathrm{i}} \mathrm{v}\right), \mathrm{d}\left(\mathrm{A}_{\mathrm{i}} \mathrm{u}, \mathrm{T}_{\mathrm{i}} \mathrm{v}\right)\right\} \forall \mathrm{i}$

$=\max \{\mathrm{d}(\mathrm{u}, \mathrm{v}), \mathrm{d}(\mathrm{u}, \mathrm{u}), \mathrm{d}(\mathrm{v}, \mathrm{v}), \mathrm{d}(\mathrm{u}, \mathrm{v}), \mathrm{d}(\mathrm{u}, \mathrm{v})\}=\mathrm{d}(\mathrm{u}, \mathrm{v})$

$\mathrm{d}(\mathrm{u}, \mathrm{v})<\mathrm{d}(\mathrm{u}, \mathrm{v})$

$=><=$ when $\mathrm{u} \neq \mathrm{v}$.

Therefore, $\mathrm{u}=\mathrm{v}$.

The proof is similar when $\mathrm{A}_{\mathrm{i}}$ and $\mathrm{S}_{\mathrm{i}} \forall \mathrm{i}$ are assumed noncompatible and $\mathrm{B}_{\mathrm{i}}$ and $\mathrm{T}_{\mathrm{i}} \forall \mathrm{i}$ are assumed continuous compatible mappings.

Hence the theorem.

Remark 3.3. If follows from the above proof that the assumption of the theorem that one of pairs, say $\left(\mathrm{B}_{\mathrm{i}}, \mathrm{T}_{\mathrm{i}}\right) \forall \mathrm{i}$ is non compatible can be weakened in the following way: There exists a sequence $\left\{\mathrm{x}_{\mathrm{n}}\right\}$ such that

$\mathrm{d}\left(\mathrm{B}_{\mathrm{i}} \mathrm{x}_{\mathrm{n}}, \mathrm{T}_{\mathrm{i}} \mathrm{x}_{\mathrm{n}}\right) \rightarrow 0 \forall \mathrm{i}$

(Equivalently, for any $\in>0, \mathrm{~B}_{\mathrm{i}}$ and $\mathrm{T}_{\mathrm{i}} \forall \mathrm{i}$ have an $\in$-coincidence point $\mathrm{x} \in$, that is $\mathrm{d}\left(\left(\mathrm{B}_{\mathrm{i}} \mathrm{x} \in, \mathrm{T}_{\mathrm{i}} \mathrm{x} \in\right)<\in \forall \mathrm{i}\right.$ ) and the sequence $\left\{\mathrm{B}_{\mathrm{i}} \mathrm{x}_{\mathrm{n}}\right\}$ is convergent[then, automatically $\left\{\mathrm{T}_{\mathrm{i}} \mathrm{x}_{\mathrm{n}}\right\} \forall \mathrm{i}$ converges].

Example3.4. Let $\mathrm{X}=[220)$ with the $\mathrm{d}$ be the usual metric on $\mathrm{X}$.

Define $A_{i}, B_{i}, S_{i}, T_{i}: X \rightarrow X, i=1,2,3, \ldots \ldots$ by

$\mathrm{A}_{\mathrm{i}} \mathrm{X}=2$ for each $\mathrm{x}$,

$\mathrm{S}_{\mathrm{i}} \mathrm{x}=\mathrm{x}$ if $\mathrm{x}<8 \mathrm{~S}_{\mathrm{i}} \mathrm{x}=8$ if $\mathrm{x}>8 \forall \mathrm{i}$

$\mathrm{B}_{\mathrm{i}} \mathrm{x}=2$, if $\mathrm{x}=2$ or $>5 \mathrm{~B}_{\mathrm{i}} \mathrm{x}=8$ if $2<\mathrm{x}<4 \mathrm{~B}_{\mathrm{i}} \mathrm{x}=3+\mathrm{x}$ if $4<\mathrm{x}<5$,

$\mathrm{T}_{\mathrm{i}} 2=2 \mathrm{~T}_{\mathrm{i}} \mathrm{x}=12+\mathrm{x}$ if $2<\mathrm{x}<4 \mathrm{~T}_{\mathrm{i}} \mathrm{x}=9+\mathrm{x}$ if $4<\mathrm{x}<5 \mathrm{~T}_{\mathrm{i}} \mathrm{x}=\mathrm{x}-3$ if $\mathrm{x}>5$ :

Then $\mathrm{A}_{\mathrm{i}}, \mathrm{B}_{\mathrm{i}}, \mathrm{S}_{\mathrm{i}}$ and $\mathrm{T}_{\mathrm{i}} \forall \mathrm{i}$ satisfy all the conditions of the above theorem and have a unique common fixed point $x=2$. It may be noted in this example that $A_{i}$ and $S_{i} \forall i$ are continuous compatible mappings while $\mathrm{B}_{\mathrm{i}}$ and $\mathrm{T}_{\mathrm{i}} \forall \mathrm{i}$ are non-compatible point wise R-weakly commuting mappings. $\mathrm{B}_{\mathrm{i}}$ and $\mathrm{T}_{\mathrm{i}} \forall \mathrm{i}$ are point wise R-weakly commuting since they commute at their coincidence points. To see that $\mathrm{B}_{\mathrm{i}}$ and $\mathrm{T}_{\mathrm{i}} \forall \mathrm{i}$ are noncompatible, let us consider a decreasing sequence $\left\{\mathrm{x}_{\mathrm{n}}\right\}$ in $\mathrm{X}$ such that $\mathrm{x}_{\mathrm{n}} \rightarrow 5$. Then $\mathrm{B}_{\mathrm{i}} \mathrm{x}_{\mathrm{n}}=2, \forall \mathrm{i}$

$\mathrm{T}_{\mathrm{i}} \mathrm{x}_{\mathrm{n}}=\mathrm{x}_{\mathrm{n}}-3 \rightarrow 2, \mathrm{~T}_{\mathrm{i}} \mathrm{B}_{\mathrm{i}} \mathrm{x}_{\mathrm{n}}=\mathrm{T}_{\mathrm{i}} 2=2 \forall \mathrm{i}$

and $\mathrm{B}_{\mathrm{i}} \mathrm{T}_{\mathrm{i}} \mathrm{x}_{\mathrm{n}}=\mathrm{B}_{\mathrm{i}}\left(\mathrm{x}_{\mathrm{n}}-3\right)=8 . \forall \mathrm{i}$ Hence $\mathrm{B}_{\mathrm{i}}$ and $\mathrm{T}_{\mathrm{i}} \forall \mathrm{i}$ are noncompatible. $\mathrm{A}_{\mathrm{i}}, \mathrm{B}_{\mathrm{i}}, \mathrm{S}_{\mathrm{i}}$ and $\mathrm{T}_{\mathrm{i}} \forall \mathrm{i}$ satisfy the contractive condition (1) but do not satisfy the contractive conditions (2) and (3). To show that (1) holds observe that $\mathrm{d}\left(\mathrm{A}_{\mathrm{i}} \mathrm{x} \mathrm{B}_{\mathrm{i}} \mathrm{y}\right)=0$ for $\mathrm{y}=2$ or $>5, \forall \mathrm{i}$ and $\mathrm{d}\left(\mathrm{A}_{\mathrm{i}} \mathrm{x} \mathrm{B}_{\mathrm{i}} \mathrm{y}\right)<\mathrm{d}\left(\mathrm{B}_{\mathrm{i}} \mathrm{y} \mathrm{T}_{\mathrm{i}} \mathrm{y}\right)<\mathrm{M}_{\mathrm{ii}}(\mathrm{x}$ y) if $2<\mathrm{y}<5 . \forall \mathrm{i}$

To show that condition (2) is not satisfied, put $\mathrm{x}=8$ and $\mathrm{y}_{\mathrm{n}}=5-1 / \mathrm{n}$. Then

$\mathrm{d}\left(\mathrm{A}_{\mathrm{i}} 8, \mathrm{~B}_{\mathrm{i}} \mathrm{y}_{\mathrm{n}}\right)=1+\mathrm{y}_{\mathrm{n}} \rightarrow 6$ and $\mathrm{M}_{\mathrm{ii}}\left(8, \mathrm{y}_{\mathrm{n}}\right)=6, \forall \mathrm{i}$ and we see that $\phi(\mathrm{t})$ cannot be defined at $\mathrm{t}=6$. Therefore, (2) does not hold.

Hence condition (3) is not satisfied either, because, as shown in [3], conditions (2) and (3) are equivalent. In fact, the function $\delta(\in)$ of condition (3) is also undefined at $\in=6$. To see this, let $\mathrm{x}=8, \mathrm{y}_{\mathrm{n}}=2+1 / \mathrm{n}$, then $\mathrm{d}\left(\mathrm{A}_{\mathrm{i}} 8, \mathrm{~B}_{\mathrm{i}} \mathrm{y}_{\mathrm{n}}\right)=6$ and $\mathrm{M}_{\mathrm{ii}}\left(8, \mathrm{y}_{\mathrm{n}}\right)=6+1 / \mathrm{n}$, and hence $\delta(\in)$ satisfying (3) cannot be defined at $\in=6$. 


\section{References}

[1]. R.P.Pant, R-weak commutativity and common fixed points, Soochow journal of mathematics 25 (1999) 37-42.

[2]. R. P. Pant, Common fixed points of weakly commuting mappings, Math. Student, 62(1993), 97-102.

[3]. J. Jachymski, Common fixed point theorems for some families of maps, Indian J. Pure Appl. Math., 25(1994), 925-937.

[4]. G. Jungck, Compatible mappings and common fixed points, Internat. J. Math. Math. Sci., 9(1986), $771-779$.

[5]. R. P. Pant, Common fixed points of noncommuting mappings, J. Math. Anal. Appl.,

[6]. 188 (1994), 436-440.

[7]. R. P. Pant, Common fixed points of sequences of mappings, Ganita, 47(1996),43-49.

[8]. G. Jungck, Compatible mappings and common fixed points, Internat. J. Math. Math. Sci., 9 (1986), $771-779$. 